Gynäkologe 2018·51:397-402

https://doi.org/10.1007/s00129-018-4240-6

Online publiziert: 27. April 2018

(c) Der/die Autor(en) 2018

\section{Redaktion}

M. Kiechle, München

W. Jonat, Kiel

A. Quante, München

CrossMark

\author{
Anne S. Quante ${ }^{1}$ Brigitte Strahwald ${ }^{2} \cdot$ Christine Fischer $^{3} \cdot$ Marion Kiechle $^{1}$ \\ ${ }^{1}$ Frauenklinik und Poliklinik, Klinikum rechts der Isar, Technische Universität München, München, \\ Deutschland \\ ${ }^{2}$ Institut für Medizinische Informationsverarbeitung, Biometrie und Epidemiologie, Ludwig-Maximilians- \\ Universität, München, Deutschland \\ ${ }^{3}$ Institut für Humangenetik, Universität Heidelberg, Heidelberg, Deutschland
}

\title{
Individualisiertes Brustkrebsrisiko - wie berechnen, wie bewerten und wie besprechen?
}

\section{Hintergrund}

Brustkrebs ist die mit Abstand häufigste Krebserkrankung der Frau [1]. In Deutschland wurde 2005 ein bundesweites Früherkennungsprogramm eingeführt. Durch das Programm soll Brustkrebs frühzeitig erkannt werden, um die Heilungschancen zu erhöhen, die Krankheitslast $\mathrm{zu}$ reduzieren und die Mortalität zu senken. Das Mammographie-Screeningprogramm steht seit einiger Zeit in der Kritik - Nutzen und Risiken werden sowohl in Fachkreisen als auch in der Öffentlichkeit heftig diskutiert [2-4]. Als Teilnahmekriterium wird nur das Alter der Frauen berücksichtigt, dabei beeinflussen weitere Risikofaktoren das Erkrankungsrisiko [5]. Das Programm wird daher dem individuell unterschiedlichen Bedarf an Früherkennung nicht gerecht. Bei vielen Frauen wird eine Mammographie ohne eindeutigen Nutzen durchgeführt. Andere Frauen, vor allem jüngere, werden trotz vorhandener Risikofaktoren nicht im Programm berücksichtigt. Die Herausforderung besteht daher darin, die Zielgruppe für die Früherkennung besser zu definieren [6].

Für eine Zielgruppe, die sog. Hochrisikofrauen, wird das risikoadaptierte Screening in Deutschland bereits angewandt. Sie haben familiär bedingt ein deutlich höheres Risiko an Brustkrebs zu erkranken als die Allgemeinbevölke- rung. Diese Frauen benötigen umfangreichere Maßnahmen der Früherkennung, da das reguläre Mammographie-Screening aufgrund des frühen Erkrankungsalters nicht ausreichend ist. Frauen, deren Familien die Einschlusskriterien des Deutschen Konsortiums für familiären Brust- und Eierstockkrebs (GC-HBOC) erfüllen und die eine Mutation in einem der bekannten Krebsgene tragen, können am intensivierten Nachsorge- bzw. Früherkennungsprogramm teilnehmen. Entscheidend ist der Nachweis einer pathogenen Mutation in den Hochrisikogenen, BRCA1, BRCA2, CDH1 oder TP53 bzw. in den moderat penetranten Genen CHEK2, PALB2, RAD51C/D, NBN oder ATM [7].

Eine besondere Herausforderung besteht jedoch, wenn in der Familie keine Veränderung in bekannten Krebsgenen gefunden wird. Die intensivierte Früherkennung wird auch dann angeboten, wenn Frauen mit auffallender Familienanamnese eine Keimbahnmutation eines bislang unbekannten Gens (Heterozygotenrisiko $>20 \%$ ) oder ein Lebensrisiko $>30 \%$ aufweisen [8]. Die Risiken werden mit einem standardisierten Risikoberechnungsverfahren - derzeit Cyrillic - kalkuliert. Dieses Verfahren ist jedoch inzwischen wissenschaftlich und technisch überholt. Erste Auswertungen der damit generierten Daten konnten zudem zeigen, dass eine Subgruppe von Frauen nicht von dieser intensivierten Früherkennung profitiert.

Mittlerweile sind neuere Risikomodelle verfügbar, die weitere Risikofaktoren für Brustkrebs basierend auf wissenschaftlichen Erkenntnissen berücksichtigen. Sie können künftig eine individualisierte Brustkrebsfrüherkennung - das „Mammographie-Screening 2.0“ - für alle Frauen ermöglichen. Dies setzt voraus, dass die Zielgruppen für ein stratifiziertes
Tab. 1 Prospektive Validierungsstudien

\begin{tabular}{|ll}
\hline Publikation & Risikomodelle \\
\hline Amir et al. (2003) [19] & Gail, Claus, IBIS, Ford \\
\hline Quante et al. (2012) [5] & Gail, IBIS \\
\hline McInnis et al. (2013) [20] & BOADICEA \\
\hline Laitman et al. (2013) [21] & BOADICEA, IBIS \\
\hline Powell et al. (2014) [22] & IBIS, Gail, BRCAPRO \\
Quante et al. (2015) [23] & IBIS, BOADICEA \\
\hline Evans et al. (2016) [24] & Mod. Claus-Tabellen, Gail, IBIS, BOADICEA \\
\hline IBIS International Breast Cancer Intervention Study, BOADICEA Breast and Ovarian Analysis of \\
Disease Incidence and Carrier Estimation Algorithm
\end{tabular}




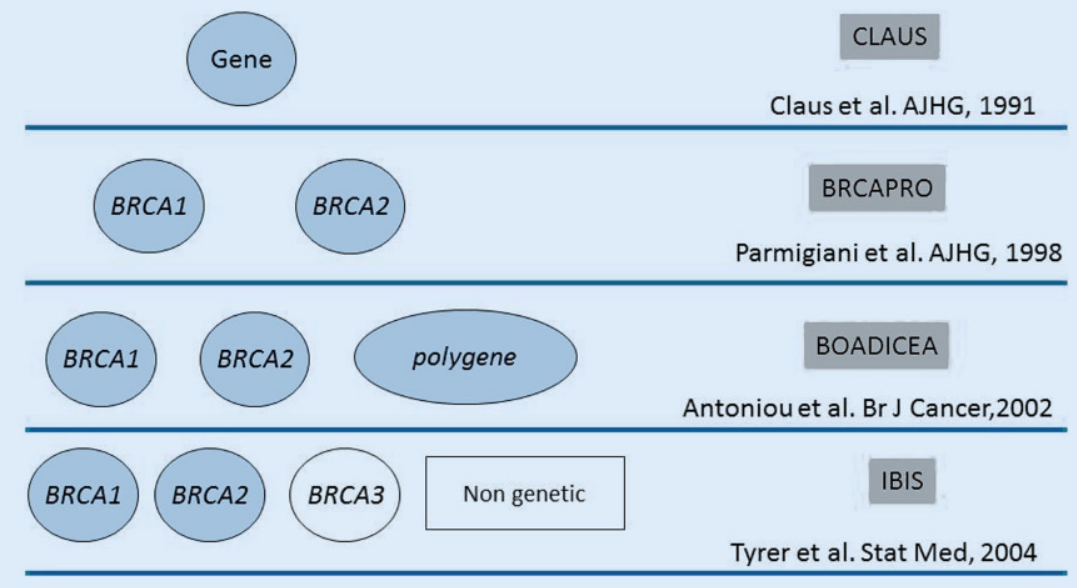

Abb. 1 ॥ Übersicht genetischer Risikomodelle für Brustkrebs (mit freundl. Genehmigung der Autoren)

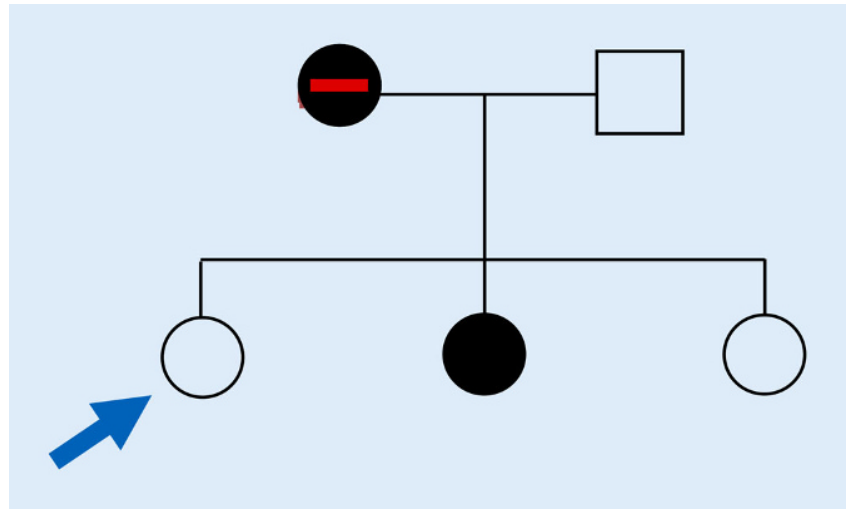

Abb. $2 \triangleleft$ Gesunde Ratsuchende aus BRCA negativer Familie (mit freundl. Genehmigung der Autoren)

Früherkennungsprogramm in Deutschland so definiert werden können, dass Frauen mit erhöhtem Risiko häufiger und Frauen mit niedrigem Risiko möglicherweise seltener untersucht werden. Ziel ist dabei, das Mammographie-Screeningprogramm effizienter zu gestalten und ein besseres Risiko-Nutzen-Profil für die teilnehmenden Frauen zu erzielen [6].

\section{》) Nutzen und Risiken} des MammographieScreeningprogramms werden nach wie vor heftig diskutiert

Eine große Herausforderung für die „Mammographie 2.0“ ist der Umgang mit den Risikomodellen. Die sichere Anwendung im Rahmen eines risikoadaptierten Screenings setzt voraus, dass die Grundlagen der Risikoprädiktion verstanden werden. Das individuelle Brustkrebsrisiko muss adäquat berechnet, bewertet und besprochen werden.

\section{Wie berechnen?}

Die Risikoberechnung basiert auf Risikomodellen für Brustkrebs. Ein Risikomodell ist ein statistisches Modell, das die Wahrscheinlichkeit für eine momentan gesunde Frau mit spezifischen Risikofaktoren (z. B. Familiengeschichte, Mutationsstatus, nichtgenetische Faktoren) berechnet, in einem vorgegebenen Zeitintervall (z. B. innerhalb von 10 Jahren oder lebenslang) an Brustkrebs zu erkranken. Es gibt 2 Arten von Risikoberechnungsmodellen, genetische und empirische [9].

Genetische Modelle basieren auf der Auswertung von Familienstudien und Segregationsanalysen. Es werden Informationen des Stammbaums (Brust- und Eierstockkrebs, Erkrankungsalter, Al- tersstruktur der Gesunden) verwendet, um mithilfe der Bayes-Formel sowie der Penetranzen altersabhängige $\mathrm{Mu}$ tations- und Erkrankungsrisiken für alle Familienangehörige eines Stammbaums zu berechnen [9]. Als erstes wurde das Claus-Modell [10] als EinGen-Modell entwickelt. Es entstand, als die Hauptgene BRCA 1 und BRCA2 noch nicht bekannt waren, und modelliert ein autosomal-dominantes Gen mit altersabhängigen Penetranzen [11]. Nach der Identifikation von BRCA1 [12] und BRCA2 [13] wurde das BRCAPRO-Modell entwickelt, das als Zwei-Gen-Modell BRCA1 und BRCA2 berücksichtigt [14]. $\mathrm{Da}$ die beiden Gene die familiäre Häufung nicht vollständig erklären konnten, wurden aktuellere Modelle mit einer polygenen Komponente entwickelt:

- BOADICEA (Breast and Ovarian

Analysis of Disease Incidence and

Carrier Estimation Algorithm; [15]) und

- IBIS (International Breast Cancer Intervention Study; [16]).

Das IBIS-Modell ist zurzeit das einzige genetische Modell, das auch nichtgenetische Risikofaktoren berücksichtigt. Das aktuellste BOADICEA-Modell bezieht weitere Testergebnisse (BRCA1, BRCA2, ATM, CHEK2, PALB2) und die Tumorpathologie in die Risikoberechnung mit ein ([17]; • Abb. 1).

Empirische Risikomodelle beruhen auf Auswertungen epidemiologischer Studien. Basierend auf Fall-Kontroll-Studien werden Risikofaktoren für Brustkrebs identifiziert und mittels logistischer Regression modelliert. Die Familienanamnese wird dabei nur in zusammengefasster Form berücksichtigt [9]. Das am meisten verwendete empirisches Risikomodell ist das Gail-Modell [18]. Es wurde auf Basis der Daten einer Fall-KontrollStudie mit etwa 6000 Probandinnen aus den USA entwickelt. Das Risiko einer Frau, innerhalb einer gewissen Zeitspanne an Brustkrebs zu erkranken, wird im Modell basierend auf einer Reihe nichtgenetischer Risikofaktoren berechnet. Dazu gehören Alter, Ethnie, Anzahl von an Brustkrebs erkrankten Verwandten ersten Grades, Anzahl vorhergehender 
Biopsien, Alter bei der Menarche und bei der ersten Lebendgeburt $[5,18]$.

Da die Brustkrebsrisiken all dieser empirischen und genetischen Programme abweichend sind, ist die Wahl des Risikoberechnungsverfahrens sehr entscheidend für die Anwendung des risikoadaptierten Screenings. Eine wichtige Voraussetzung ist, dass die Modelle an einer unabhängigen prospektiven Kohorte validiert wurden. Dabei ist entscheidend, wie gut die Modelle kalibriert sind (d.h. inwiefern weichen beobachte und erwartete Fälle in Untergruppen voneinander ab) und wie gut sie diskriminieren, d.h. Erkrankte von Nichterkrankten unterscheiden können. Wichtige prospektive Validierungsstudien der o.g. Risikoberechnungsmodelle sind in - Tab. $1 \mathrm{zu}$ sammengefasst.

\section{Wie bewerten?}

Für das individualisierte Brustkrebsscreening werden Frauen zunächst entsprechend ihrer Risikokonstellation in Risikogruppen eingeteilt, anschließend wird ein stratifiziertes Früherkennungsprogramm angeboten. Allerdings gibt es derzeit keinen international einheitlichen Grenzwert für den Bereich, ab dem Frauen der Hochrisikogruppe zugeteilt werden

Für eine gesunde Ratsuchende aus einer Familie, welche die Einschlusskriterien für eine genetische Testung erfüllt, bei deren betroffenen Familienangehörigen (Index) jedoch keine Veränderung in einem der bisher bekannten Brustkrebsgenen gefunden wurde, richtet sich die Empfehlung der intensivierten Früherkennung nach dem errechneten Erkrankungsrisiko (• Abb. 2). In Deutschland wird dieses Risiko momentan mit Cyrillic berechnet. Allen Frauen, bei denen ein Lebensrisiko $>30 \%$ oder Heterozygotenrisiko $>20 \%$ vorliegt, wird ab dem 30. Lebensjahr die intensivierte Früherkennung einschließlich MRT (Magnetresonanztomographie) angeboten $[7,8]$. Da das Cyrillic-Risikoberechnungsverfahren inhaltlich und technisch als überholt gilt, gibt es Bestrebungen, es durch modernere Verfahren abzulösen. Der Wechsel auf ein anderes Risikoberechnungsverfahren hat jedoch zur

Gynäkologe 2018·51:397-402 https://doi.org/10.1007/s00129-018-4240-6

(c) Der/die Autor(en) 2018

\section{A. S. Quante · B. Strahwald · C. Fischer · M. Kiechle}

\section{Individualisiertes Brustkrebsrisiko - wie berechnen, wie bewerten und wie besprechen?}

\section{Zusammenfassung}

Das Mammographie-Screeningprogramm steht seit einiger Zeit in der Kritik. Als Teilnahmekriterium wird nur das Alter der Frauen berücksichtigt, dabei beeinflussen weitere Risikofaktoren das Erkrankungsrisiko. Neuere Risikomodelle für Brustkrebs berücksichtigen einige dieser Faktoren. Sie ermöglichen eine individualisierte Brustkrebsfrüherkennung - das "Mammographie-Screening 2.0". Hierdurch soll ermöglicht werden, dass Frauen mit erhöhtem Risiko häufiger und Frauen mit niedrigem Risiko möglicherweise seltener untersucht werden. Ziel ist es, das Mammographie-Screeningprogramm effizienter zu gestalten und Frauen eine bessere Risiko-Nutzen-Bewertung zu ermöglichen. Eine große Herausforderung ist dabei der Umgang mit den Risikomodellen. Die sichere Anwendung im Rahmen eines risikoadaptierten Screenings setzt voraus, dass die Grundlagen der Risikoprädiktion verstanden werden. Das individuelle Brustkrebsrisiko muss adäquat berechnet, bewertet und besprochen werden.

Schlüsselwörter

Mammographie - Mammaneoplasien . Krebsfrüherkennung · Kommunikation . Gemeinsame Entscheidungsfindung

\section{Individualized risk of breast cancer-How should it be calculated, evaluated and discussed?}

\section{Abstract}

There has been a lot of controversy about the mammography screening program. Screening recommendations for breast cancer in the general population are currently based solely on the age of an individual, despite the fact that additional genetic and non-genetic factors are known to influence cancer risk. Recent breast cancer risk models include these factors. They allow the implication of a risk-adapted screening approach, "mammography screening 2.0". On the one hand there is a need to intensify diagnostic procedures for women at higher risk but on the other hand it is desirable to avoid unnecessary diagnostic procedures in women who are unlikely to develop breast cancer. The aim is to improve the efficiency of the screening program and to help guide screening decisions by including patients' individual risk profiles and preferences; however, the implementation of such a strategy faces new challenges, such as the particular prediction model to use, the ways to evaluate and to communicate risks in the screening context.

\section{Keywords}

Mammography · Breast neoplasms - Early detection of cancer - Communication - Shared Decision making
Folge, dass sich auch die berechneten Brustkrebsrisiken und Mutationswahrscheinlichkeiten für die Frauen ändern könnten und damit auch die Zielgruppe für das Früherkennungsprogramm. Nach den NCCN(National Comprehensive Cancer Network)-Guidelines [25] wird einer Frau in den USA mit einem Lebensrisiko $>20 \%$ die intensivierte Früherkennung einschließlich MRT ab 30 Jahre angeboten. Es wird jedoch nicht angeben, welches Risikomodell verwendet werden soll. Eigene Auswertungen an einer prospektiven Kohorte von über 1700 Frauen zeigten, dass die Ergebnisse von BOADICEA und IBIS bezüglich des Lebensrisikos stark voneinander abweichen. Bei Verwendung der NCCN-Guidelines stufte das IBIS-Modell etwa $60 \%$ der Probandinnen in die Hochrisikogruppe ein, bei BOADICEA nur etwa $20 \%$ [23]. Nach den NICE(National Institute for Health and Care Excellence)-Guidelines [26] in England wird einer Frau mit einem Lebensrisiko ab $30 \%$ oder einem Zehnjahresrisiko ab $8 \%$ eine intensivierte Früherkennung einschließlich MRT angeboten. So werden in England sehr viel weniger Frauen in ein intensiviertes 

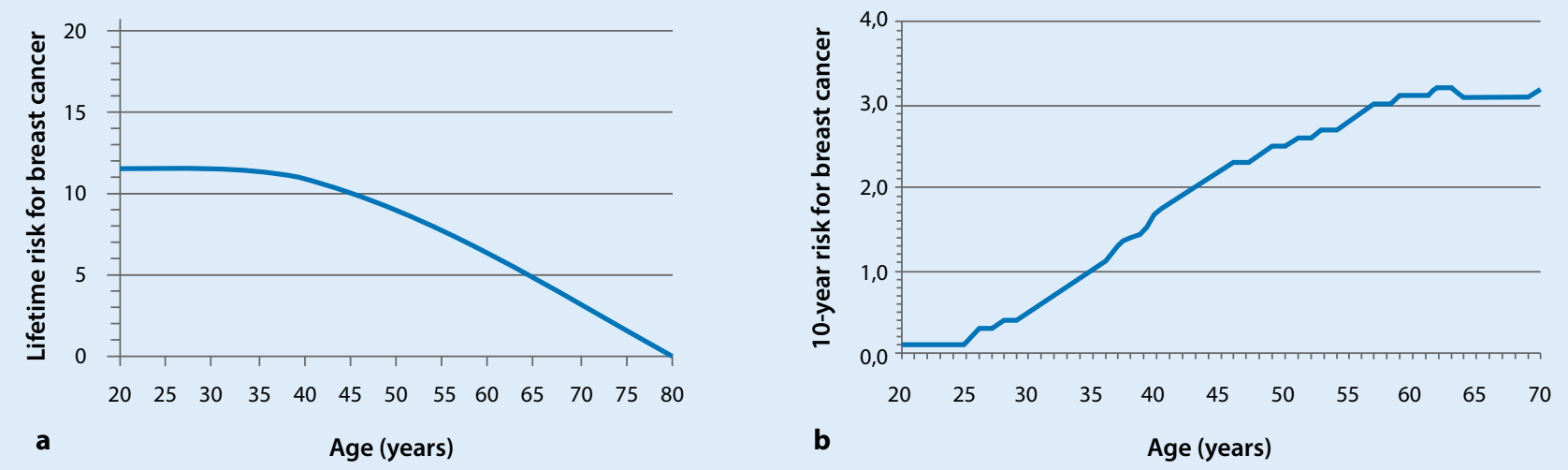

Abb. $3 \Delta$ a Lebensrisiko für Frauen in Abhängigkeit vom Alter.bZehnjahresrisiko fürFrauen in Abhängigkeit vom Alter (Daten freundlich zur Verfügung gestellt vom Robert Koch Institut per Mail am 21.07.2016; mit freundl. Genehmigung der Autoren)

Früherkennungsprogramm aufgenommen als in Deutschland oder in den USA. Allerdings werden nur in NICE-Guidelines auch Frauen mit einem moderat erhöhten Risiko (Lebensrisiko 17-30\% bzw. Zehnjahresrisiko 3-8\%) berücksichtigt und können das Früherkennungsangebot ohne MRT ab 40 Jahren wahrnehmen.

Die Regeln im internationalen Vergleich beziehen sich sowohl auf das Lebensrisiko als auch auf das Risiko, innerhalb der nächsten 10 Jahre Brustkrebs zu entwickeln (Zehnjahresrisiko). Dies führt jedoch zu unterschiedlichen „Hochrisiko"-Zielgruppen: - Abb. 3a zeigt das durchschnittliche Lebensrisiko für Frauen vom aktuellen Alter bis zu einem Alter von 80 Jahren. Definiert man den Grenzwert z.B. bei $20 \%$ Lebensrisiko werden eher jüngere Frauen als „Hochrisiko" klassifiziert. Die • Abb. 3b zeigt das Zehnjahresrisiko für Frauen in Abhängigkeit vom Alter, es steigt mit dem Alter an. Definiert man den Grenzwert z. B. bei 3,34\% Zehnjahresrisiko, werden eher ältere Frauen als "Hochrisiko" klassifiziert.

Für das risikoadaptierte Screening ist es insgesamt sinnvoll, kürzere Intervalle von 10 Jahren zu verwenden, um die Risiken zu ermitteln. Wir konnten zeigen, dass die Abweichungen zwischen Modellen z. B. IBIS und BOADICEA für das Zehnjahresrisiko geringer sind als für das Lebensrisiko [23]. Dies liegt u. a. daran, dass verschiedene Modelle das Lebensrisiko unterschiedlich definieren: entwe- der bis zum Alter von 80 Jahren oder bis zu 85 Jahren. Hinzu kommt, dass sich prospektive Validierungsstudien stets auf das Zehnjahresrisiko beziehen, nie auf das Lebensrisiko. Die Beobachtungszeit reicht nicht aus um das Lebensrisiko zu evaluieren [23].

Eine Grundvoraussetzung für das risikoadaptierte Screening ist, dass Grenzwerte festgelegt werden. Nur so können die teilnehmenden Frauen in Risikogruppen eingeteilt werden, um auf dieser Basis eine klinische Konsequenz für die Früherkennung abzuleiten. Die Entscheidung, welches Risikomodell der Berechnung zugrunde liegt, ist enorm weitreichend, da die Ergebnisse unterschiedlich ausfallen.

\section{I) Eine Umstellung des Risikoberechnungsverfahrens birgt eine große Herausforderung}

Eine Umstellung des Risikoberechnungsverfahrens birgt daher eine große Herausforderung. Die Gruppe der Frauen, die fortan das Angebot einer intensivierten Früherkennung erhält, verändert sich dadurch. Dieser Aspekt bedarf einer besonders sorgfältigen Kommunikation und Aufklärung. Es ist daher unbedingt erforderlich, dass alle beteiligten Berufsgruppen die Hintergründe der Umstellung verstehen, das neue Risikoberechnungsverfahren anwenden und die Ergebnisse kompetent kommunizieren.

\section{Wie besprechen?}

Schon die Kommunikation im Rahmen des bisherigen, "normalen“ Mammographie-Screenings ist eine Herausforderung. Die Frauen sollen in die Lage versetzt werden, eine informierte Entscheidung für oder gegen die Teilnahme zu treffen [27]. Dieser Informed Consent setzt voraus, dass Nutzen und Risiken inhaltlich verstanden, richtig interpretiert und auf die eigene Situation angewandt werden können. Die dafür nötige Gesundheitskompetenz bzw. Health Literacy umfasst u.a. auch ein Grundverständnis von Statistik. Die eigentliche Entscheidung sollte idealerweise gemeinsam im Sinne eines Shared Decision Making getroffen werden.

Dieser Anspruch ist allerdings oft nur schwer zu erfüllen. Die statistischen Aussagen über positive und negative Auswirkungen des Screenings werden sowohl von Ärzten als auch von ratsuchenden Laien häufig fehlinterpretiert [28]. Der Nutzen wird zum Teil massiv überschätzt, während die Risiken eher bagatellisiert werden [29].

\section{I) Statistische Aussagen über Screeningeffekte werden von Ärzten wie von Laien häufig fehlinterpretiert}

Bei der Einführung oder Umstellung eines risikoadaptierten Screenings muss 
diese Ausgangssituation ganz besonders beachtet werden. Um die Höhe des individuellen Brustkrebsrisikos zu berechnen wird ein komplexes statistisches Modell verwenden. Das Ergebnis ist ein Schätzwert, kein Messergebnis, es beinhaltet daher auch ein unterschiedlich hohes Maß an Unsicherheit. Damit die teilnehmenden Frauen dieses Ergebnis richtig verstehen und einordnen können, sollten die behandelnden Ärzte es umfassend erklären können - was wiederum ein tiefes Verständnis der Grundlagen voraussetzt. Dies kann nicht vorausgesetzt werden, daher sollten bedarfsgerechte Informationsangebote und Praxishilfen $\mathrm{zu}$ den Risikoberechnungen entwickelt werden. Weitere Angebote sollten auch die Grundlagen der Risikokommunikation und des Shared Decision Making vermitteln.

Generell gehen viele Ärzte davon aus, dass sie bereits eine gemeinsame Entscheidungsfindung praktizieren. Untersuchungen legen nahe, dass die Einschätzung trügt und ein „perception-reality gap“ vorliegt [30]. Dies ist umso problematischer, als bei der Umstellung des Risikoberechnungsverfahrens besonders 2 Subgruppen vor einer schwierigen Situation stehen. Die Frauen in der einen Subgruppe wurden nach dem bisherigen Verfahren nicht als „Hochrisiko“ eingestuft, nach dem neuen Verfahren - und dem neuen Schwellenwert - ändert sich dies. Die betroffenen Frauen müssen daher verstehen, worauf die Änderung von "Nichthochrisiko“ in „Hochrisiko“ beruht, um dann entscheiden zu können, ob und wie sie an einer intensivierten Früherkennung teilnehmen wollen.

》) Nur informierte Ärzte können
richtig aufklären und eine
partizipative Entscheidung
ermöglichen

Noch problematischer ist die Situation für Frauen in der anderen Subgruppe. Sie galten bislang als „Hochrisiko“ und erhalten nun die Information, dass sie nicht mehr in diese Kategorie eingeordnet werden. Voraussichtlich werden die Reaktionen darauf sehr unterschiedlich ausfal- len, je nach individueller Risikowahrnehmung. Diese Risk Perception wiederum wird von ärztlicher Seite aus oft nicht wahrgenommen, obwohl sie einen maßgeblichen Effekt auf gesundheitsbezogene Entscheidungen hat.

Um eine adäquate Kommunikation und ein Shared Decision Making für das risikoadaptierte Screening zu etablieren, müssen daher begleitende Angebote entwickelt werden, um die Ärzte zu unterstützen. Nur informierte Ärzte können die Frauen richtig über das Verfahren aufklären und eine partizipative Entscheidung ermöglichen.

\section{Fazit für die Praxis}

- Das Mammographie-Screening sieht ein risikoadaptiertes Screening vor, das weitere bekannte Risikofaktoren für Brustkrebs miteinbezieht.

- Es gibt in Deutschland bereits eine Zielgruppe von „Hochrisiko"-Frauen, denen ein intensiviertes Früherkennungsprogramm einschließlich MRT-Untersuchungen angeboten wird. Dieses Programm ist unumstritten bei Mutationsträgerinnen, jedoch kontrovers bei nur rechnerischer Hochrisikosituation (mehr als $20 \%$ iger Mutationswahrscheinlichkeit oder $30 \%$ iges lebenslanges Brustkrebsrisiko, berechnet mit der Software Cyrillic). Hier muss das Berechnungsverfahren dringend aktualisiert werden. Wichtig ist, dass die Zielgruppen künftig besser definiert werden können.

- Die rechtliche Grundlage für die Verwendung von Risikomodellen ist derzeit in der Diskussion (CE Mark) und muss noch geklärt werden.

- Auch aus ökonomischer Sicht ist zu prüfen, ob die individualisierte Screening-Mammographie kosten-/ nutzeneffizienter ist.

\section{Korrespondenzadresse

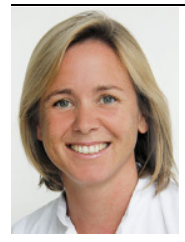 \\ Dr. med. A. S. Quante, MPH \\ Frauenklinik und Poliklinik, Klinikum rechts der Isar, Technische Universität München Ismaninger Str. 22, 81675 München, Deutschland anne.quante@tum.de}

\section{Einhaltung ethischer Richtlinien}

Interessenkonflikt. A.S. Quante, B. Strahwald, C. Fischer und M. Kiechle geben an, dass kein Interessenkonflikt besteht.

Dieser Beitrag beinhaltet keine von den Autoren durchgeführten Studien an Menschen oder Tieren.

Open Access. Dieser Artikel wird unter der Creative Commons Namensnennung 4.0 International Lizenz (http://creativecommons.org/licenses/by/4.0/deed. de) veröffentlicht, welche die Nutzung, Vervielfältigung, Bearbeitung, Verbreitung und Wiedergabe in jeglichem Medium und Format erlaubt, sofern Sie den/die ursprünglichen Autor(en) und die Quelle ordnungsgemäßnennen, einen Link zur Creative Commons Lizenz beifügen und angeben, ob Änderungen vorgenommen wurden.

\section{Literatur}

1. Quante AS, Ming C, Rottmann M et al (2016) Projections of cancer incidence and cancer-related deaths in Germany by 2020 and 2030. Cancer Med 5:2649-2656

2. Gøtzsche P, Jørgensen K (2013) Screening for breast cancer with mammography (Review). Cochrane Database Syst Rev. https://doi.org/10. 1002/14651858.CD001877.pub5

3. Independent UK Panel on Breast Cancer Screening (2012) The benefits and harms of breast cancer screening: an independent review. Lancet 380:1778-1786

4. Lauby-Secretan B, Scoccianti C, Loomis D et al (2015) Breast-Cancer Screening - viewpoint of the IARC working group. NEngl J Med 372:2353-2358

5. Quante AS, Whittemore AS, Shriver T et al (2012) Breast cancer risk assessment across the risk continuum: genetic and nongenetic risk factors contributing to differential model performance. Breast Cancer Res 14:R144

6. Quante A, Kiechle M (2017) Symposium „Brustkrebs-Screening 2.0: Personalisiert - Prädiktiv Präventiv-Partizipatorisch? Geburtshilfe Frauenheilkd 77:960-962

7. Schmutzler R (2017) Konsensusempfehlung des Deutschen Konsortiums Familiärer Brust- und Eierstockkrebs zum Umgang mit Ergebnissen der Multigenanalyse. Geburtshilfe Frauenheilkd 77:733-739

8. Kiechle M (2016) Brustkrebsfrüherkennung Zielgruppen, Methoden, Nutzen und Nebenwirkungen. Onkologe 22:550-557 
9. Bickeböller H, Fischer C (2007) Einführung in die genetische Epidemiologie. Springer, Berlin, Heidelberg

10. Claus EB, Risch N, Thompson WD (1994) Autosomal dominant inheritance of early-onset breast cancer. Implicationsfor risk prediction. Cancer 73:643-651

11. Engel CZS, Fischer C (2015) Familärer Brustkrebs empirische Erkrankungsrisiken und Risikoberechnungsmodelle. medgen 27:217-222

12. Miki Y, Swensen J, Shattuck-Eidens D et al (1994) A strong candidate for the breast and ovarian cancer susceptibility gene BRCA1. Science 266:66-71

13. Tavtigian SV, Simard J, Rommens J et al (1996) The complete BRCA2 gene and mutations in chromosome 13q-linked kindreds. Nat Genet 12:333-337

14. Parmigiani G, Berry D, Aguilar O (1998) Determining carrier probabilities for breast cancersusceptibility genes BRCA1 and BRCA2. Am J Hum Genet 62:145-158

15. Antoniou AC, Pharoah PD, McMullan G et al (2002) A comprehensive model for familial breast cancer incorporating BRCA1, BRCA2 and other genes. $\mathrm{Br} J$ Cancer 86:76-83

16. Tyrer J, Duffy SW, Cuzick J (2004) A breast cancer prediction model incorporating familial and personal risk factors. Stat Med 23:1111-1130

17. Lee AJ, Cunningham AP, Tischkowitz Met al (2016) Incorporating truncating variants in PALB2, CHEK2, and ATM into the BOADICEA breast cancer risk model. Genet Med 18:1190-1198

18. Gail MH, Brinton LA, Byar DP et al (1989) Projecting individualized probabilities of developing breast cancer for white females who are being examined annually. J Natl Cancer Inst 81:1879-1886

19. Amir E, Evans DG, Shenton A et al (2003) Evaluation of breast cancer risk assessment packages in the family history evaluation and screening programme. J Med Genet 40:807-814

20. Maclnnis RJ, Bickerstaffe A, Apicella $C$ et al (2013) Prospective validation of the breast cancer risk prediction model BOADICEA and a batchmode version BOADICEACentre. Br J Cancer 109:1296-1301

21. Laitman Y, Simeonov M, Keinan-Boker Let al (2013) Breast cancer risk prediction accuracy in Jewish Israeli high-risk women using the BOADICEA and IBIS risk models. Genet Res (Camb) 95:174-177

22. Powell M, Jamshidian F, Cheyne K et al (2014) Assessing breast cancer risk models in Marin County, a population with high rates of delayed childbirth. Clin Breast Cancer 14:212-220.e211

23. Quante AS, Whittemore AS, Shriver T et al (2015) Practical problems with clinical guidelines for breast cancer prevention based on remaining lifetime risk. J Natl Cancer Inst 107:djv124

24. Evans DGR, Warwick J, Astley SM et al (2012) Assessing individual breast cancer risk within the UK National Health Service Breast Screening Program: a new paradigm for cancer prevention. Cancer Prev Res (Phila) 5:943-951

25. Bevers TB, Anderson BO, Bonaccio E et al (2009) NCCN clinical practice guidelines in oncology: breast cancer screening and diagnosis. J Natl Compr Canc Netw 7:1060-1096

26. Evans DG, Graham J, O'Connell S et al (2013) Familial breast cancer: summary of updated NICE guidance. BMJ 346:f3829

27. Stefanek ME (2011) Uninformed compliance or informed choice? A needed shift in our approach to cancer screening. J Natl Cancer Inst 103:1821-1826

28. Wegwarth O, Gigerenzer G (2018) The barrier to informed choice in cancer screening: statistical illiteracy in physicians and patients. Recent Results Cancer Res 210:207-221

29. Hoffmann TC, Del Mar C (2015) Patients' expectations of the benefits and harms of treatments, screening, and tests: a systematic review. JAMA Intern Med 175:274-286

30. Stiggelbout AM, Van der Weijden T, De Wit MP et al (2012) Shared decision making: really putting patients at the centre of healthcare. BMJ 344:e256

\section{Jetzt neu}

Nature Reviews PrimeViews

\section{Als Poster zum Herausnehmen}

In der Zeitschrift Nature Reviews erscheinen sog. Disease Primers - globale Übersichten zu einer bestimmten Krankheit und noch offenen Schlüsselfragen. Aufgebaut sind die Artikel modular und decken Epidemiologie, Krankheitsmechanismen, Diagnostik, Therapie, Screening und Prävention ab. Jede Woche werden neue Artikel von einem internationalen Autorenteam bestehend aus Wissenschaftlern, translationalen und klinischen Forschern publiziert. Jeder Artikel wird begleitet von einem PrimeView. Die PrimeViews sind illustrierte, kurze Zusammenfassungen der Disease Primers in Form von Postern, produziert von dem Editorial Team. Auf den Postern wird jeweils die Originalpublikation angegeben. Dort findet man sowohl die Übersichten, als auch die PrimeViews selbst zum Lesen, Downloaden oder Teilen.

Für das Jubiläumsjahr 2018 wurden inhaltlich passende Themen ausgesucht und übersetzt und liegen einzelnen Ausgaben als Poster zum Herausnehmen bei.

In dieser Ausgabe finden Sie das Poster zum Thema "Ovarialkarzinom".

Viel Freude und gute Lektüre wünscht Innen die Redaktion von Der Gynäkologe.

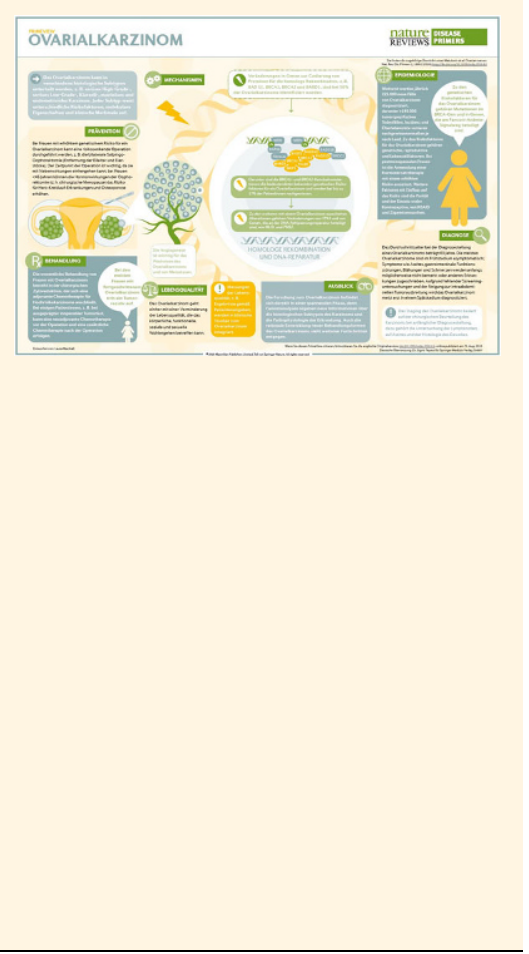

